\title{
Can FDG PET/CT monitor the response to hormonal therapy in breast cancer patients?
}

\author{
Laura Evangelista $\cdot$ Domenico Rubello • \\ Giorgio Saladini
}

Published online: 20 December 2011

(C) Springer-Verlag 2011

As breast cancer becomes metastatic, it becomes incurable and the goals of treatment are palliation of symptoms, prolongation of survival, and maintenance or improvement of the quality of life. The median survival of women with metastatic disease is approximately 2 years, but it may be longer than 10 years in some patients with slowly progressive skeletal or nodal metastases. Advanced breast cancer is mostly treated with endocrine therapies or cytostatic agents, or palliative radiotherapy/systemic metabolic radiotherapy in those with painful bone metastases and ulcerative tumours. Hormonal therapy is a cancer treatment that removes hormones or blocks their action and stops cancer cells growing. Endocrine therapy has often been employed in breast cancer patients as neoadjuvant or adjuvant treatment, significantly improving the outcomes in women with breast cancer positive for oestrogen receptors (ER), while in women with ER- negative cancer, systemic chemotherapy remains the best choice for decreasing disease recurrence and death. Hormonal drugs produce their effects in various ways: (1) by blocking the effect of a specific enzyme, (2) by suppressing hormone production, and (3) by inactivating the target receptors. A number of pharmacological agents have been developed that modulate tumour cell ER function or reduce the levels of circulating oestrogens. Among these compounds are the selective ER modulators (SERMs;

\footnotetext{
L. Evangelista $(\bowtie) \cdot$ G. Saladini

Radiotherapy and Nuclear Medicine Unit, Istituto Oncologico Veneto (IOV - IRCCS),

Via Gattamelata, 64,

35128 Padova, Italy

e-mail: laura.evangelista@tin.it

D. Rubello

Department of Nuclear Medicine, Radiology, Medical Physics, 'Santa Maria della Misericordia' Hospital,

Rovigo, Italy

tamoxifen, raloxifen), pure antioestrogens (fulvestrant/Faslodex), luteinizing hormone-releasing hormone agonists (leuprolide, goserelin), and third-generation selective aromatase inhibitors (AIs; anastrozole/Arimidex, letrozole/ Femara, exemestane/Aromasin). Tamoxifen acts as an antioestrogen against breast cancer cells through competitive inhibition of oestrogen binding to the ER and inhibition of the expression of oestrogen-modulated genes. The result is a slowing of cell proliferation and therefore the arrest in G1 phase of the proliferative process. AIs act by inhibiting the cytochrome P-450 enzyme that promotes the conversion of androstenedione and testosterone to oestrone and oestradiol, respectively, resulting in a fall in circulating oestrogens to very low levels.

Diagnostic imaging tools are able to assess response to hormonal therapy, but it remains unclear how to evaluate the impact of treatment on visceral and nonvisceral metastases. ${ }^{18}$ F-FDG PET imaging has been demonstrated to play an important role in the management of patients with breast cancer, particularly in the assessment of tumour response to chemotherapy. Most published studies have assessed breast cancer response to neoadjuvant treatment [1-8] and interim chemotherapy evaluation (after the first and second cycle [1-3]), and have demonstrated a high correlation with pathological response or predicted outcome in patients with metastatic disease. In the present issue of the European Journal of Nuclear Medicine and Molecular Imaging, Mortazavi-Jehanno et al. [9] report the results in 22 patients with recurrent or metastatic breast cancer at diagnosis who underwent serial FDG PET/CT before and after $8 \pm 2$ weeks after the start of hormonal therapy. Of these 22 patients, 12 showed disseminated disease (multiorgan), 7 had a single organ lesion and 3 had a single metastasis (bone and lymph node involvement). In a high percentage of patients nonvisceral involvement (68\% bone metastases, and 50\% 
lymph node metastases) was found, whereas $14 \%$ of metastases involved the lung and 5\% the liver. Exclusive hormonal therapy was started, including AI in $73 \%$ and antioestrogens in $23 \%$ of patients. EORTC criteria were used to define the metabolic response, distinguishing patients with a partial metabolic response from patients with stable or progressive metabolic disease. Patients with a partial metabolic response and stable metabolic disease had a significantly longer disease-free survival than patients with progressive metabolic disease (log rank test, $p<$ 0.0001 ), whereas the overall survival was similar among the groups (log rank test, $p=0.603$ ). Therefore, progressive metabolic disease was associated with a poor prognosis, underlining the utility in this patient subset of adding a new treatment, such as immunological therapy or chemotherapy.

Some authors have already analysed the support of FDG PET in monitoring the response to treatment in metastatic breast cancer, but they focused their attention on primary chemotherapy rather than hormonal therapy. Gennari et al. [10] evaluated 13 patients with visceral metastasis of breast cancer who underwent epirubicin/paclitaxel combination chemotherapy as first-line treatment. The authors found a significant decrease in tumour FDG uptake soon after the first cycle in responders, and on the contrary found no modification of FDG uptake during treatment in nonresponders. Schwarz et al. [11] performed a prospective study in patients with metastatic breast cancer. Sequential ${ }^{18} \mathrm{~F}$-FDG PET allowed prediction of response to treatment after the first cycle of chemotherapy (epirubicin/cyclophosphamide and epirubicin/paclitaxel). The use of changes in the standardized uptake value (SUV; $20 \%$ decrease) obtained by serial ${ }^{18} \mathrm{~F}$-FDG PET studies as a surrogate for monitoring therapy response offered improved patient care by individualizing treatment and avoiding ineffective chemotherapy. Couturier et al. [12] prospectively enrolled 20 patients with metastatic breast cancer who underwent anthracycline-based, taxane-based and Herceptin chemotherapy. Sequential PET studies were used to monitor responses to chemotherapy. Using SUV as the metabolic feature, PET was unable to correctly evaluate response after one cycle alone, but it added some clinical information after three cycles of chemotherapy.

Except for the uncommon clinical flare reaction that may occur 7-10 days after induction of hormonal therapy, tumour response to endocrine therapy, as assessed by clinical, laboratory and imaging examinations, is generally delayed by several months after induction. Several studies have shown that the metabolic flare reaction, corresponding to an increase in tumour FDG uptake 7-10 days after the start of hormonal therapy, is predictive of response to such treatment $[13,14]$, but data are lacking about the delayed effect of hormonal therapy on tumour metabolism. As reported by Mortazavi-Jehanno et al. [9], PET/CT allows evaluation of the delayed response to hormonal therapy and can provide prognostic data on the metabolic response to therapy.

In bone-dominant disease, in which morphological modalities do not allow assessment of tumour response [15] and in which hormonal therapy plays a major role, tumour FDG uptake can be used as a surrogate marker to monitor early response to therapy $[15,16]$. Du et al. [15] considered 25 patients with bone metastatic breast cancer who underwent sequential FDG PET/CT studies for monitoring responses to treatment. The authors investigated the morphological changes on CT and FDG uptake after treatment in order to evaluate responses to chemotherapy or hormonal therapy. Of the 25 patients, 8 who received hormonal therapy alone showed a reduction in FDG uptake on sequential PET scans when the therapy was proven to be effective. The findings suggest that FDG uptake reflects the activity of bone metastases in breast cancer independently of morphological characteristics, whereas the radiographic changes which varied among individual patients could not be correlated with the presence of active tumour. Tateishi et al. [16] retrospectively analysed 102 patients with bone metastatic breast cancer. Of these 102 patients, 76 had received chemotherapy plus hormonal therapy and the remainder had received chemotherapy alone. The metabolic responses to therapy were evaluated in terms of the reduction in SUV and total lesion glycolysis, and thus treatment responses were calculated in relation to the semiquantitative PET values. The authors found that a decrease in SUV after treatment was an independent predictor of response duration in patients with metastatic breast cancer with bone metastases. There were important differences among the three studies discussed in their approaches to testing the responses to treatment: FDG uptake in the first study [15], change in SUV and total lesion glycolysis between two consecutive PET scans in the second study [16], and EORTC criteria in the study by Mortazavi-Jehanno et al. [9]. The assessment of metabolic response with FDG PET has not yet been standardized and a joint effort is needed to address this. This represents a challenge not only for breast cancer but also for other tumours.

In the study by Mortazavi-Jehanno et al. [9], ER-positive and HER2-negative breast cancer was one of the inclusion criteria. Both immunohistochemical features determine the so-called luminal breast cancer that, based on the Ki67 value $(<20 \%$ or $>20 \%)$, can be divided into luminal A or luminal $\mathrm{B}$, the latter being associated with a worse prognosis. In a recent study by Cottu et al. [17], the response of eight luminal breast cancer xenografts to hormonal therapy was evaluated, and they were found to demonstrate oestradioldependence. Oestrogen removal from the diet and therapeutic oestrogen deprivation by ovariectomy or letrozole significantly inhibited the effect on tumour growth.

Furthermore, Mortazavi-Jehanno et al. [9] found no link between the metabolic response to hormonal therapy and 
CA-15.3, an assay that is associated with a false-negative rate of $26 \%$. This observation underlines the fact that the serum CA-15.3 assay as the only criterion for the assessment of therapy response in patients with metastatic breast cancer during active therapy cannot recommended and must be associated with diagnostic imaging [18], history and physical examination [19]. Sonoo and Kurebayashi suggested that serial levels of tumour markers taken during therapy might not always correlate with therapy response [20]. The authors observed an initial increase within the first 3 months and a subsequent decrease in tumour marker levels in up to one-third of patients experiencing clinical benefit. One reason for this spike phenomenon might be possible tumour flair associated with the partial agonist properties of SERMs and for fulvestrant, as demonstrated by Bartsch et al. [21]. This latter study suggested that increased CA-15.3 levels may be observed in patients experiencing a partial response with fulvestrant, but it may be seen in both stable and progressive disease. Thus, an increase in tumour markers should not be taken as a definite sign of de novo progression without radiological verification.

Mortazavi-Jehanno et al. also pose a clinical and imaging question (a question also raised by Linden et al.): among the current available biomarkers which one is more relevant to predict response to endocrine therapy by molecular imaging?. Radiochemistry has enriched the radiopharmaceutical field with many types of tracers, most of them already employed in the clinical setting. Markers of proliferation $\left({ }^{18} \mathrm{~F}\right.$-fluorothymidine), of metabolism $\left({ }^{18} \mathrm{~F}\right.$-FDG, ${ }^{11} \mathrm{C}$-methionine, ${ }^{11} \mathrm{C} /{ }^{18} \mathrm{~F}$-choline, ${ }^{18} \mathrm{~F}$-dopa) and receptor-like $\left({ }^{18} \mathrm{~F}\right.$-fluoroethyloestradiol) can be used for different indications. In 2006, Linden et al. [22] evaluated the response to endocrine therapy with ${ }^{18} \mathrm{~F}$-fluorooestradiol (FES) PET. Enrolled in the study were 47 patients with recurrent or metastatic breast cancer and the indication endocrine therapy, and the response to treatment was evaluated with FES PET. FES PET provided incremental predictive value over standard clinical selection criteria in a challenging and heavily pretreated patient population. Lindholm et al. [23] studied 13 patients with metastatic breast cancer. In all patients, ${ }^{11} \mathrm{C}$-methionine (MET) PET before and after polychemotherapy (chemotherapy and endocrine therapy) was performed for the assessment of response to treatment. In their pilot study, the authors found that MET PET seemed to be useful especially in the thoracic area for identifying new areas of recurrence and its uptake clearly diminished in breast cancer metastases after successful therapy.

The determination of ER expression by immunohistochemistry is essential to select the most appropriate therapy. However, ER determination may be limited by sampling errors, heterogeneity in receptor level in the primary tumour, and the absence of ER expression in metastatic and/or recurrent sites, leading to discordance [22]. These conditions can explain why only $30-40 \%$ of patients with ER-positive breast cancer respond objectively to endocrine therapy. The resistance to endocrine therapy may be for intrinsic reasons or due to acquired resistance following prolonged use. Furthermore, endocrine therapy is associated with vasomotor symptoms (tamoxifen), musculoskeletal discomfort (AI) and occasionally more serious side effects (thrombosis and endometrial cancer from tamoxifen or osteoporotic fracture from AIs). These problems can affect overall quality of life and even reduce life expectancy $[2,24]$. Identifying predictors of endocrine responsiveness is therefore important to avoid unnecessary toxicity and to promote the selection of alternative treatment strategies in patients with endocrine-resistant tumours [1]. As reported by Lindstrom at the 2011 European Multidisciplinary Cancer Congress, cancer expression of ER can change with time (change rate $30 \%$ ).

The association of FES PET for the noninvasive evaluation of ER expression in recurrent and metastatic breast cancer with FDG PET for monitoring response to hormonal therapy could be a favourable conjunction to obtain the main objectives of endocrine therapy: disease stabilization and the best and prolonged palliation.

\section{References}

1. Smith IC, Welch AE, Hutcheon AW, Miller ID, Payne S, Chilcott F, et al. Positron emission tomography using [(18)F]-fluorodeoxyD-glucose to predict the pathologic response of breast cancer to primary chemotherapy. J Clin Oncol. 2000;18(8):1676-88.

2. Shelling M, Avril N, Nahrig J, Kuhn W, Romer W, Sattler D, et al. Positron emission tomography using [(18)F]fluorodeoxyglucose for monitoring primary chemotherapy in breast cancer. J Clin Oncol. 2000;18:1689-95.

3. Honkoop AH, van Diest PJ, de Jong JS, Linn SC, Giaccone G, Hoekman K, et al. Prognostic role of clinical, pathological and biological characteristics in patients with locally advanced breast cancer. Br J Cancer. 1998;77(4):621-6.

4. Berriolo-Riedinger A, Touzery C, Riedinger JM, Toubeau M, Coudert B, Arnould L, et al. [18F]FDG-PET predicts complete pathological response of breast cancer to neoadjuvant chemotherapy. Eur J Nucl Med Mol Imaging. 2007;34(12):1915-24.

5. Jansson T, Westli J, Ahlström H, Lilja A, Långström B, Bergh J. Positron emission tomography studies in patients with locally advanced and/or metastatic breast cancer: a method for early therapy evaluation? J Clin Oncol. 1995;13(6):1470-7.

6. Dunnwald LK, Gralow J, Ellis GK, Livingston RB, Linden HM, Specht JM, et al. Tumor metabolism and blood flow changes by positron emission tomography: relation to survival in patients treated with neoadjuvant chemotherapy for locally advanced breast cancer. J Clin Oncol. 2008;26(27):4449-57.

7. Ogston KN, Miller I, Payne S, Hutcheon AW, Sarkar TK, Smith I, et al. A new histological grading system to assess response of breast cancers to primary chemotherapy: prognostic significance and survival. Breast. 2003;12(5):320-7.

8. Veronesi U, De Cicco C, Galimberti VE, Fernandez JR, Rotmensz $\mathrm{N}$, Viale G, et al. A comparative study on the value of FDG-PET and sentinel node biopsy to identify occult axillary metastases. Ann Oncol. 2007;18:473-8. 
9. Mortazavi-Jehanno N, Giraudet AL, Champion L, Lerebours F, Le Stanc E, Edeline V, et al. Assessment of response to endocrine therapy using FDG PET/CT in metastatic breast cancer: a pilot study. Eur J Nucl Med Mol Imaging. 2011. doi:10.1007/s00259-011-1981-z.

10. Gennari A, Donati S, Salvadori B, Giorgetti A, Salvadori PA, Sorace O, et al. Role of 2-[18F]-fluorodeoxyglucose (FDG) positron emission tomography (PET) in the early assessment of response to chemotherapy in metastatic breast cancer patients. Clin Breast Imaging. 2000;1(2):156-61.

11. Schwarz JD, Bader M, Jenicke L, Hemminger G, Jänicke F, Avril $\mathrm{N}$. Early prediction of response to chemotherapy in metastatic breast cancer using sequential 18F-FDG PET. J Nucl Med. 2005;46(7):1144-50.

12. Couturier O, Jerusalem G, N'Guyen JM, Hustinx R. Sequential positron emission tomography using $18 \mathrm{~F}$ fluorodeoxyglucose for monitoring response to chemotherapy in metastatic breast cancer. Clin Cancer Res. 2006;12(21):6437-43.

13. Dehdashti F, Flanagan F, Morimer J, Katzenellenbogen J, Welch M, Siegel B. Positron emission tomographic assessment of "metabolic flare" to predict response of metastatic breast cancer to antiestrogen therapy. Eur J Nucl Med. 1999;26:51-6.

14. Mortimer J, Dehdashti F, Siegel B, Trinkaus K, Katzenellenborgen J, Welch M. Metabolic flare: indicator of hormone responsiveness in advanced breast cancer. J Clin Oncol. 2001;19:2797803.

15. Du Y, Cullum I, Illidge TM, Ell PJ. Fusion of metabolic function and morphology: sequential [18F]fluorodeoxyglucose positronemission tomography/computed tomography studies yield new insights into the natural history of bone metastases in breast cancer. J Clin Oncol. 2007;25(23):3440-7.

16. Tateishi U, Gamez C, Dawood S, Yeung HWD, Cristofanilli M, Macapinlac HA. Bone metastases in patients with metastatic breast cancer: morphologic and metabolic monitoring of response to systemic therapy with integrated PET/CT. Radiology. 2008;247 (1):189-96.

17. Cottu P, Marangoni E, Assayag F, de Cremoux P, Vincent-Salomon A, Guyader Ch, et al. Modeling of response to endocrine therapy in a panel of human luminal breast cancer xenografts. Breast Cancer Res Treat, 2011. doi:10.1007/s10549-011-1815-5.

18. Champion L, Brain E, Giraudet A, Le Stanc E, Wartski M, Edeline $\mathrm{V}$, et al. Breast cancer recurrence diagnosis suspected on tumor marker rising: value of whole-body 18FDG-PET/CT imaging and impact on patient management. Cancer. 2011;117:1621-9.

19. Harris L, Fritsche H, Mennel R, Norton L, Ravdin P, Taube S, et al. American Society of Clinical Oncology 2007: update of recommendations for the use of tumour markers in breast cancer. J Clin Oncol. 2007;25:5287-91.

20. Sonoo H, Kurebayashi J. Serum tumor markers kinetics and the clinical course of patients with advanced breast cancer. Surg Today. 1996;26:205-7.

21. Bartsch R, Wenzel C, Pluschnig U, Hussian D, Sevelda U, Altorjai $\mathrm{G}$, et al. Prognostic value of monitoring tumour markers CA 15.3 and CEA during fulvestrant treatment. BMC Cancer. 2006;6:81.

22. Linden HM, Stekhova S, Link JM, Gralow JR, Livingston RB, Ellis GK, et al. Quantitative fluoroestradiol positron emission tomography imaging predicts response to endocrine treatment in breast cancer. J Clin Oncol. 2006;24(18):2793-9.

23. Lindholm P, Lapela M, Nagrenb K, Lehikoinen P, Minn H, Jyrkkio S. Preliminary study of carbon-11 methionine PET in the evaluation of early response to therapy in advanced breast cancer. Nucl Med Commun. 2009;30:30-6.

24. Kaufmann M, von Minckwitz G, Bear H, Buzdar A, McGale P, Bonnefoi $\mathrm{H}$, et al. Recommendations from an international expert panel on the use of neoadjuvant (primary) systemic treatment of operable breast cancer: new perspectives 2006. Ann Oncol. 2007;18:1927-34. 